\title{
Quasibinary amorphous phase in a three-dimensional system of particles with repulsive-shoulder interactions
}

\author{
Yu. D. Fomin, ${ }^{1}$ N. V. Gribova, ${ }^{1}$ V. N. Ryzhov, ${ }^{1, a)}$ S. M. Stishov, ${ }^{1}$ and Daan Frenkel ${ }^{2}$ \\ ${ }_{1}^{1}$ Institute for High Pressure Physics, Russian Academy of Sciences, Troitsk 142190, Moscow Region, Russia \\ ${ }^{2}$ FOM Institute for Atomic and Molecular Physics, Amsterdam, 1009 DB. The Netherlands and \\ Department of Chemistry, University of Cambridge, Cambridge, CB2 1EW, United Kingdom
}

(Received 20 February 2008; accepted 10 July 2008; published online 13 August 2008)

\begin{abstract}
We report a computer-simulation study of the equilibrium phase diagram of a three-dimensional system of particles with a repulsive-step potential. Using free-energy calculations, we have determined the equilibrium phase diagram of this system. At low temperatures, we observe a number of distinct crystal phases. However, under certain conditions the system undergoes a glass transition in a regime where the liquid appears thermodynamically stable. We argue that the appearance of this amorphous low-temperature phase can be understood by viewing this one-component system as a quasibinary mixture. (C) 2008 American Institute of Physics.
\end{abstract}

[DOI: $10.1063 / 1.2965880]$

Many ordered materials can undergo a first-order phase transition that does not alter the symmetry of the phase. In the early work of Hemmer and Stell, ${ }^{1}$ it was proposed that such isostructural phase transitions are to be expected if the interaction between the particles in addition to the ordinary hard core has a "soft" core combined with an attractive interaction at larger distances. The work of Ref. 1 focused specifically on isostructural phase transitions in materials such as Ce or Cs, but since then many authors have studied a wide range of model system that could exhibit isostructural transitions. ${ }^{2-12}$ Most of these systems have a repulsive intermolecular potential that has a region of negative curvature, a feature that is known to be present in the interatomic potentials of some pure metallic systems, metallic mixtures, electrolytes, and colloidal systems. The simplest example of a negative-curvature potential is the repulsive-step potential which consists of a hard core plus a finite repulsive shoulder at a larger radius. Systems of particles interacting through such pair potentials can possess a rich variety of phase transitions and thermodynamic anomalies, including liquidliquid phase transitions, ${ }^{5,6}$ waterlike anomalies, ${ }^{7}$ and isostructural transitions in the solid region..$^{10,11}$

The repulsive-step potential has the form

$$
\Phi(r)=\left\{\begin{array}{lc}
\infty, & r \leqslant d \\
\varepsilon, & d<r \leqslant \sigma \\
0, & r>\sigma,
\end{array}\right.
$$

where $d$ is the diameter of the hard core, $\sigma$ is the width of the repulsive step, and $\varepsilon$ its height. In the low-temperature limit $\widetilde{T} \equiv k_{B} T / \varepsilon \ll 1$, the system reduces to a hard-sphere systems with hard-sphere diameter $\sigma$, while in the limit $\widetilde{T} \gg 1$, the system reduces to a hard-sphere model with a hard-sphere diameter $d$. For this reason, melting at high and low temperatures follows simply from the hard-sphere melting curve

${ }^{a)}$ Electronic mail: ryzhov@ hppi.troitsk.ru.
$P=c T / \sigma^{\prime 3}$, where $c \approx 12$ and $\sigma^{\prime}$ is the relevant hard-sphere diameter ( $\sigma$ and $d$, respectively). A changeover from the low- $T$ to high- $T$ melting behavior should occur for $\widetilde{T}=\mathcal{O}(1)$. The precise form of the phase diagram depends on the ratio $s \equiv \sigma / d$. For large enough values of $s$ one should expect to observe in the resulting melting curve a maximum that should disappear as $s \rightarrow 1 .^{12}$ The phase behavior in the crossover region may be very complex, as shown below.

In our simulations we have used a smoothed version of the repulsive-step potential [Eq. (1)], which has the form

$$
\Phi(r)=\left(\frac{d}{r}\right)^{n}+\frac{1}{2} \varepsilon\left(1-\tanh \left(k_{0}\left(r-\sigma_{s}\right)\right)\right)
$$

where $n=14, k_{0}=10$. We have considered the following values of $\sigma_{s}: \sigma_{s}=1.15,1.35,1.55$. In the remainder of this paper we use the dimensionless quantities: $\widetilde{\mathbf{r}} \equiv \mathbf{r} / d, \widetilde{P} \equiv P d^{3} / \varepsilon, \widetilde{V}$ $\equiv V / N d^{3} \equiv 1 / \widetilde{\rho}$. As we will only use these reduced variables, we omit the tildes.

In order to get a hint about the phase diagrams of the system, we computed energies per particle for different crystal ground states for $\sigma_{s}=1.15,1.35$. In the upper part of Fig. 1 we plot a schematic phase diagram at $T=0$ for $\sigma_{s}=1.15$. It is obtained from the calculation of the ground state energy of the face-centered cubic (fcc) and body-centered cubic (bcc) crystal structures. We also considered several other structures for this potential-simple cubic (SC), hcp, diamond structure, face-centered orthorhombic (fco), and body-centered orthorhombic (BCO) but all these were unstable.

In the lower part of Fig. 1 we show the schematic phase diagram at $T=0$ for $\sigma_{s}=1.35$. It is plotted from the calculation of the ground state energies of the fcc, bcc, SC, diamond, and FCO structures for $\sigma_{s}=1.35$. Figure 1 shows that there exists a range of densities where the stable phase has a FCO structure which unit cell is increased along the $z$ axis in 1.6 times. This is a clear indication that the phase diagrams in the solid region are quite complex. It should be also noted 


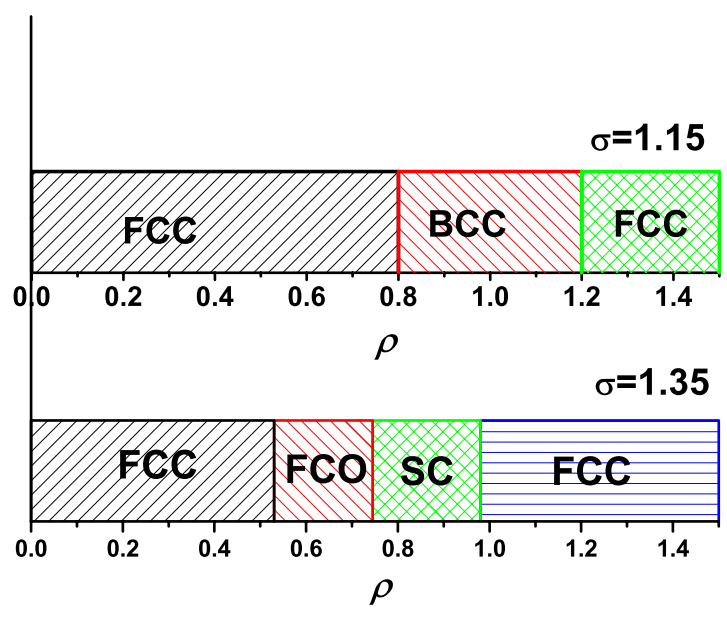

FIG. 1. (Color online) Ground state. The schematic phase diagrams at $T=0$ for $\sigma_{s}=1.15$ and $\sigma_{s}=1.35$.

that we could not compare the energy of the ideal crystalline structures with the energy of possible amorphous structure for $T=0$.

To determine the phase diagram at non-zero temperature, we performed constant- $N V T$ molecular dynamics (MD) simulations combined with free-energy calculations. In all cases, periodic boundary conditions were used. The number of particles varied between 250 and 500. No system-size dependence of the results was observed. The system was equilibrated for $5 \times 10^{6} \mathrm{MD}$ time steps. Data were subsequently collected during $3 \times 10^{6} \delta t$ where the time step $\delta t$ $=5 \times 10^{-5}$.

In some region of the phase diagram for $\sigma_{s}=1.35$, we found that the liquid phase was thermodynamically stable down to the lowest temperatures that we could probe. In this region of the phase diagram, the dynamics of the system was very sluggish, forcing us to perform much longer MD simulations (up to $90 \times 10^{6} \mathrm{MD}$ steps).

In order to map out the phase diagram of the system, we computed its Helmholtz free energy using the thermodynamic integration: the free energy of the liquid phase was computed via thermodynamic integration from the dilute gas limit, ${ }^{13}$ and the free energy of the solid phase was computed by thermodynamic integration to an Einstein crystal. ${ }^{13,14}$ In the Monte Carlo (MC) simulations of solid phases, data were collected during $5 \times 10^{4}$ cycles after equilibration. To improve the statistics (and to check for internal consistency) the free energy of the solid was computed at many dozens of different state points and fitted to multinomial function. The fitting function we used is $a_{p, q} T^{p} V^{q}$, where $T$ and $V=1 / \rho$ are the temperature and specific volume and powers $p$ and $q$ are connected through $p+q=N$. The value $N$ we used for the most of calculations is 5 . For the low-density fcc phase $\left(\sigma_{s}\right.$ =1.35) $N$ was taken 4 , since we had less data points. The transition points were determined by a double-tangent construction. Although fitting procedure could be potentially
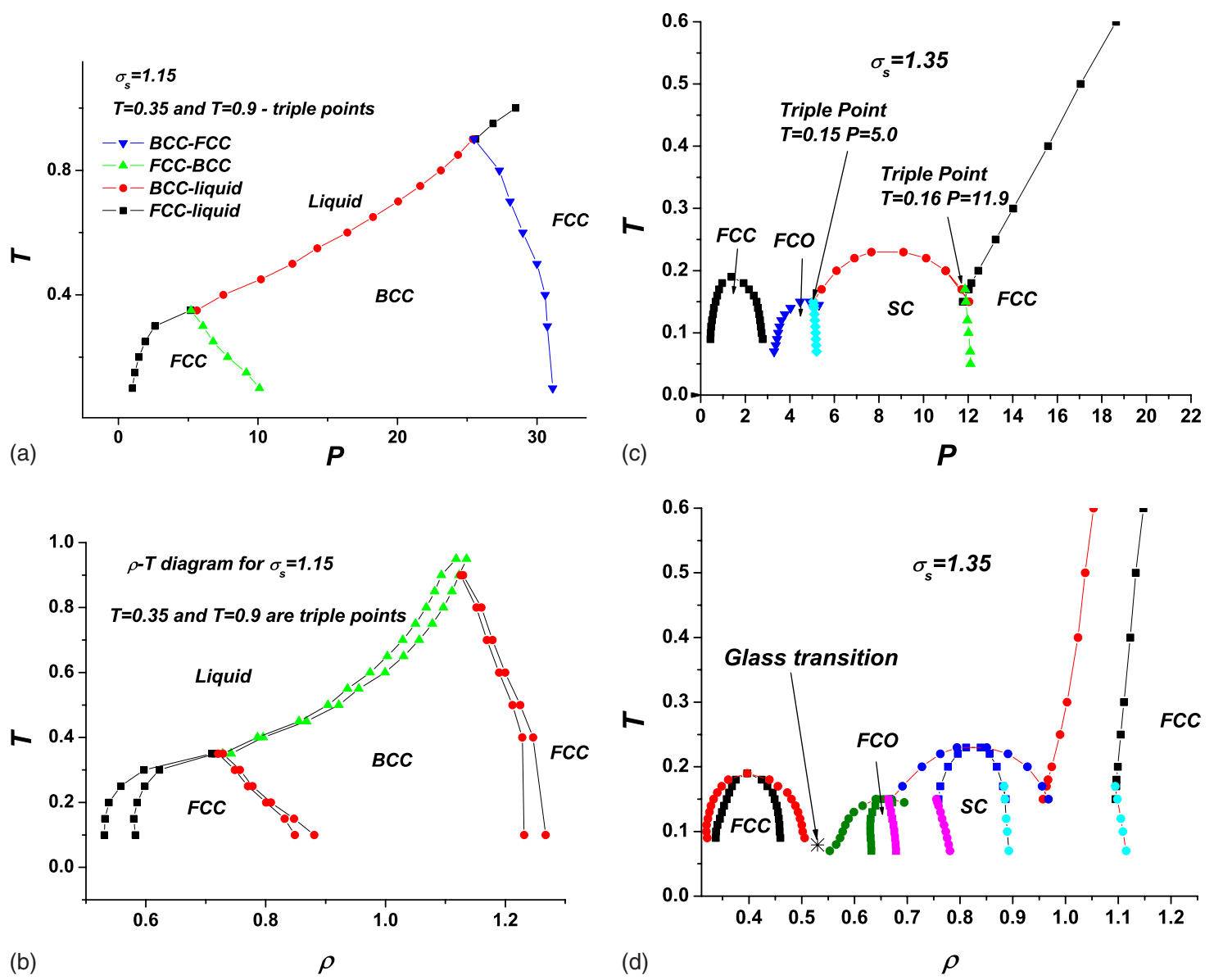

FIG. 2. (Color online) Phase diagram of the system of particles interacting through the potential (2) with $\sigma_{s}=1.15,1.35$ in $P-T$ and $\rho$ - $T$ planes. The asterisk in (d) corresponds to the glass transition. 

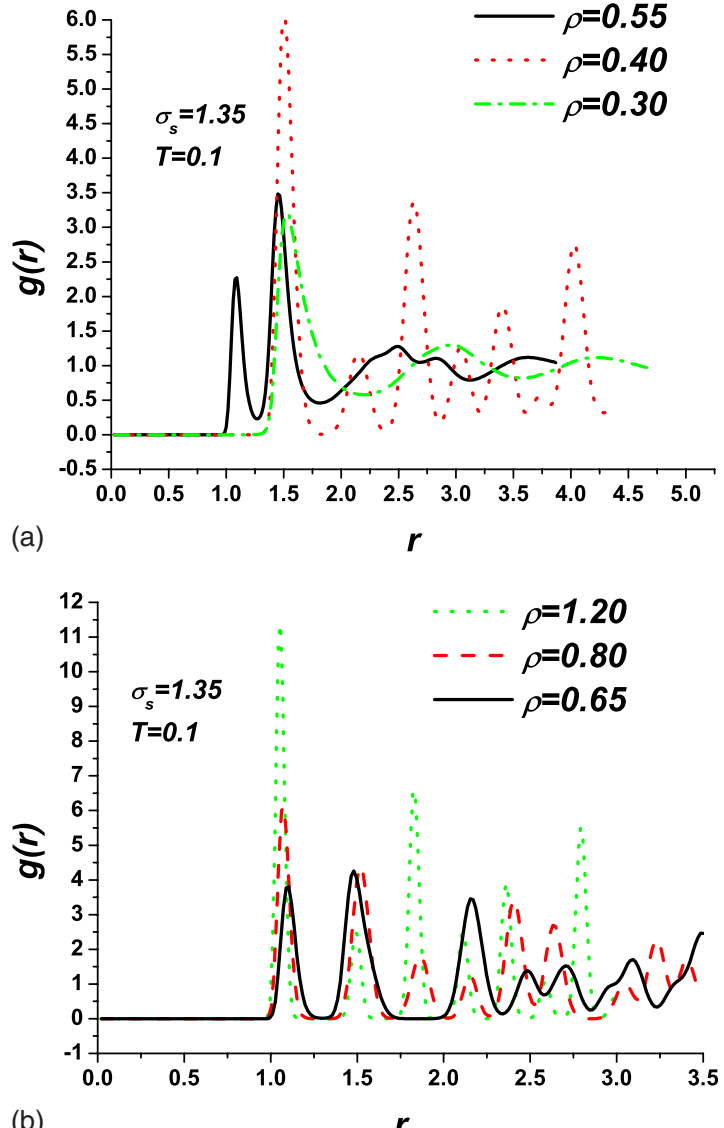

(b)

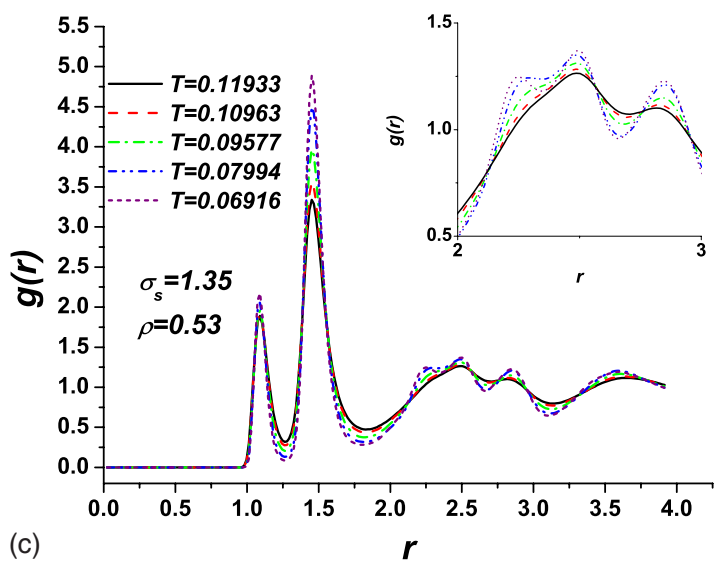

FIG. 3. (Color online) RDFs for different density ranges for $\sigma_{s}=1.35$ and $T=0.1[(\mathrm{a})$ and (b)] and (c) for different temperatures in amorphous phase $(\rho=0.53)$.

dangerous in the sense of making some errors, we believe that our fitting is correct. During our fitting we control the difference between measured and fitted free energies. The maximum difference was of the order of $10^{-3}$. Given free energies themselves of the order of 10 , we think that this is a good precision. Another test consists in differentiating the free-energy fit and comparing it to our pressure data, which also confirmed the consistency of our method.

Figure 2 shows the phase diagrams that we obtain from the free-energy calculations for two different values of $\sigma_{s}$. Figures 2(a) and 2(b) show the phase diagram of the system with $\sigma_{s}=1.15$. One can see that for the system with $\sigma_{s}$ $=1.15$ there are no maxima in the melting curve. In a soft-
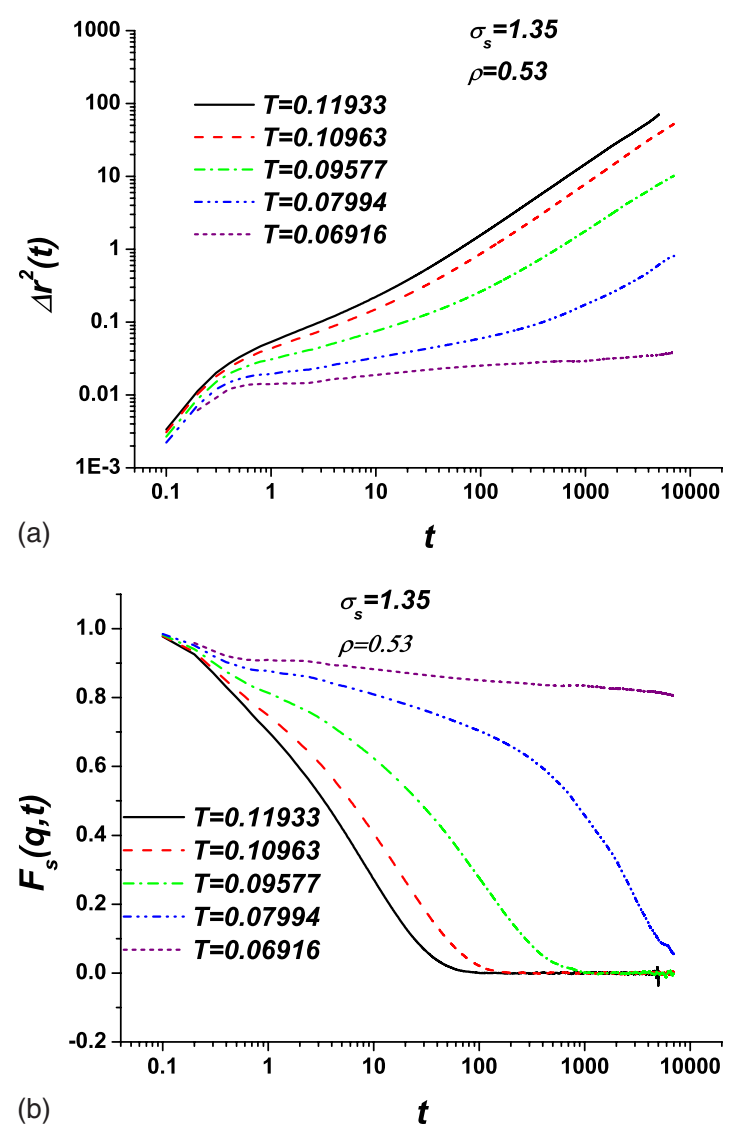

FIG. 4. (Color online) (a)MSD and (b) self-correlation function $F_{s}(q, t)$ as functions of time for $\sigma=1.35$ for different temperatures at $\rho=0.53$.

sphere system described by the potential $1 / r^{14}$ a fcc structure has been reported. ${ }^{15}$ However, the addition of a small repulsive step leads to the appearance of the fcc-bcc transition shown in Figs. 2(a) and 2(b). It should be noted that in Ref. 16 the phase diagram of the system with the potential (1) have been calculated in the framework of the densityfunctional perturbation theory for small values of the step width $\sigma$. The results for $\sigma=1.16$ are in qualitative agreement with Fig. 2(a); however, we would like to emphasize that the perturbation theory does not give the possibility to calculate the low-density phase diagram for larger values of $\sigma$ [see Figs. 2(c) and 2(d)].

Figures 2(c) and 2(d) show the phase diagram of the system with $\sigma_{s}=1.35$ in the $P-T$ and $\rho-T$ planes. There is a clear maximum in the melting curve at low densities. The phase diagram consists in two isostructural fcc parts corresponding to close packing of the small and large spheres separated by a sequence of structural phase transitions.

This complexity of the phase diagram can be understood from the shape of the potential. Since the potential is purely repulsive, the particles tend to minimize both hard- and softcore overlaps. Such overlaps can be avoided in the low pressure part of the phase diagram. Upon increasing the density, the system crystallizes into fcc phase which corresponds to the close packing of the particles at the soft core. If the pressure increases more, the particles begin to penetrate the soft core but still avoid hard-core overlaps. Hence, both parts of the potential are important now. In Fig. 3(a) we show the 


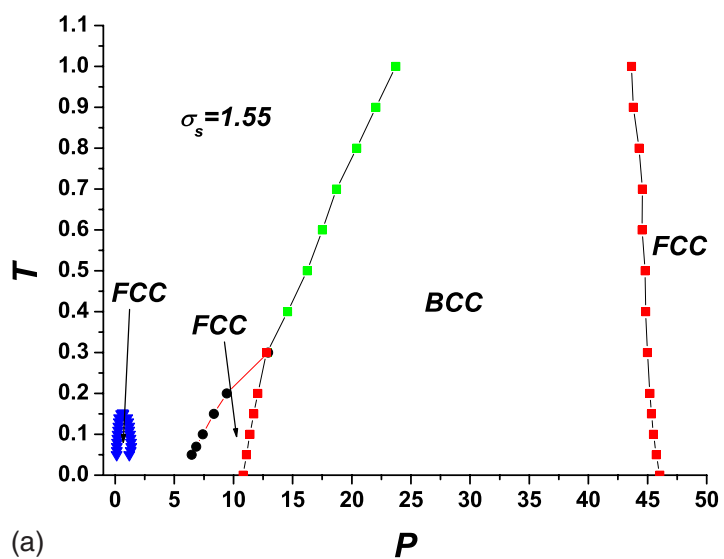

(a)

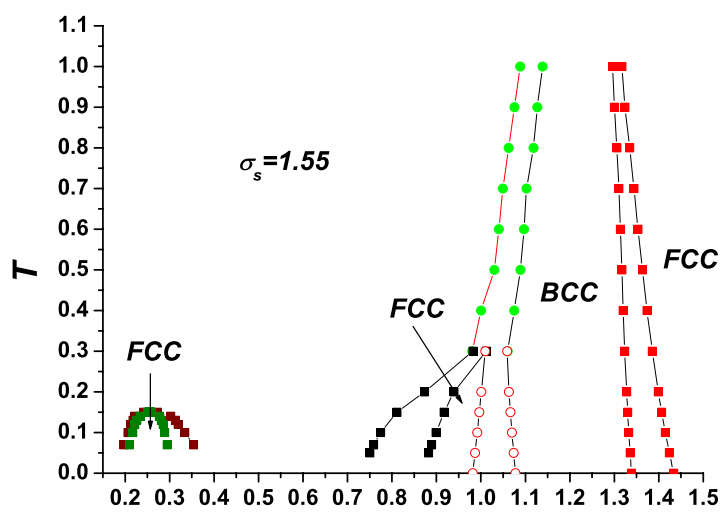

(b)

$\rho$

FIG. 5. (Color online) Phase diagram of the system of particles interacting through the potential (2) with $\sigma_{s}=1.55$ in $P-T$ and $\rho$ - $T$ planes.

behavior of the radial distribution function (RDF) in this density range. The first peak of radial distribution functions for the low-density liquid and fcc lattice corresponds to the large spheres distance, i.e., there is no overlap of soft repulsive cores. However, when the density increases a new peak develops at the hard-core distance, showing the onset of competition of two distances and, as a result, the frustration in the system. We consider the amorphous region properties in more details below.

The FCO phase corresponds to the situation when the particles are packed in $X-Y$ planes in accordance with the hard core but the planes are arranged at the soft-core distance (layered structure). As a result a particle has four nearest neighbors in this structure. If we further increase the pressure, more particles penetrate the soft core, but still the closed packed structures are not favorable. Finally, at very high pressures region we observe the fcc phase corresponding to the $r^{-14}$ limit. One can see the RDFs of these crystal structures in Fig. 3(b). Despite the difference in structures the first peak of all of them corresponds to the hard-core distance. The density in this region is already enough high to avoid frustration because the hard-core distance arrangement is more preferable.

Interestingly, there is a region of the phase diagram where we have not found any stable crystal phase. In principle it could be a drawback of our method-we suppose some crystal structures which could be stable and check their stability, so one can imagine that there are other structures
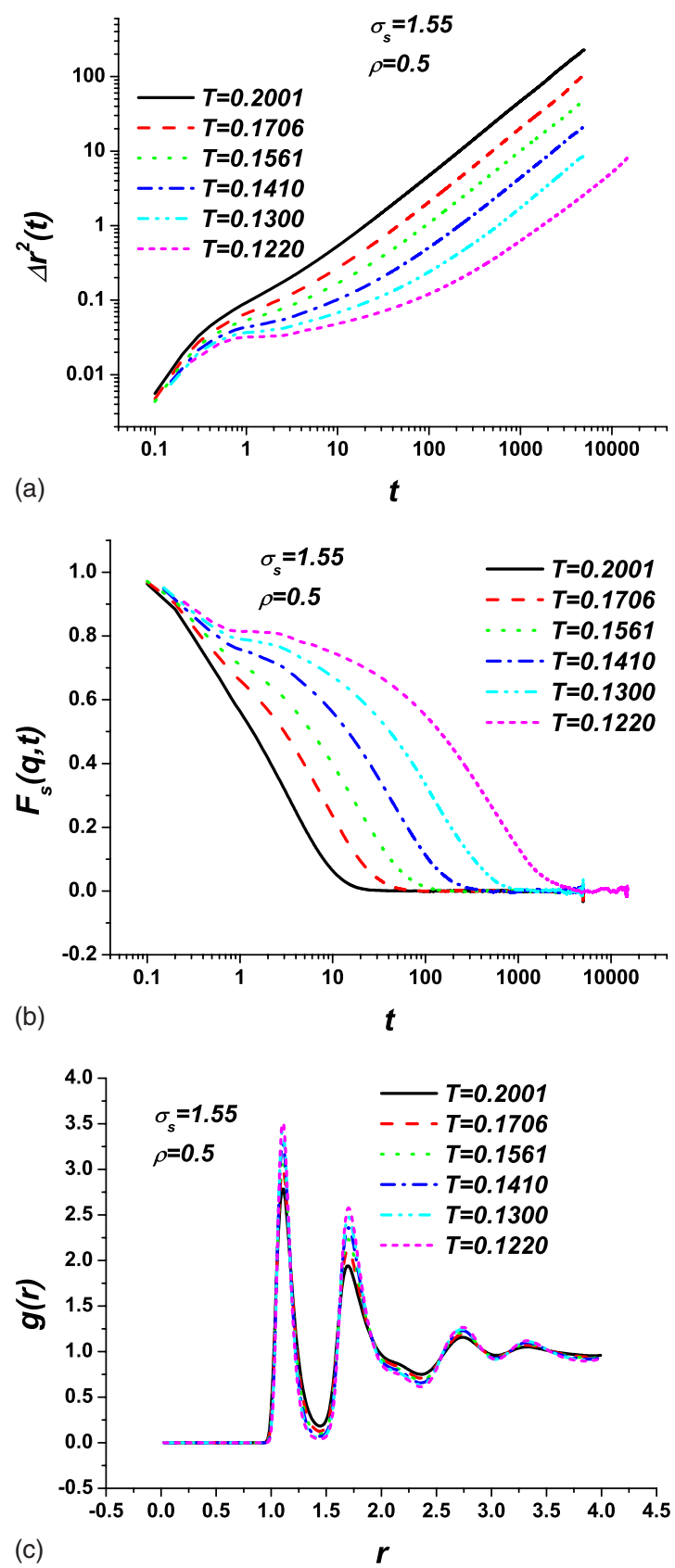

FIG. 6. (Color online) (a) RDF, (b) MSD, and (c) self-correlation function $F_{s}(q, t)$ as functions of time for $\sigma=1.55$ for different temperatures at $\rho=0.5$.

which we have not considered in our study. However, we think that no crystal structure is stable in this density range because of frustration. To check this point we calculated the RDF of the system in this region [Fig. 3(c)] for $\rho=0.53$ and different temperatures. One can see that there is no indication of any crystal structure. This behavior of RDF is typical to the glass transition. ${ }^{17-19}$ It should be noted that the ground state properties (see Fig. 1) and properties at finite temperature (Fig. 2) are consistent except for this disordered "gap" in the phase diagram because we cannot calculate the zero temperature energy of disordered phase and compare it to the free energy of crystal structures at $T=0$. However, given the instability of the crystal at rather low temperatures we believe that crystal is not stable here at any arbitrary low temperature. 


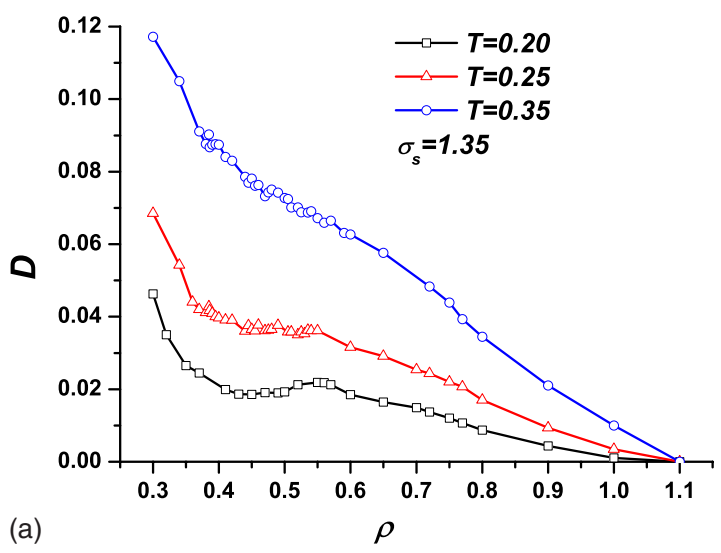

(a)

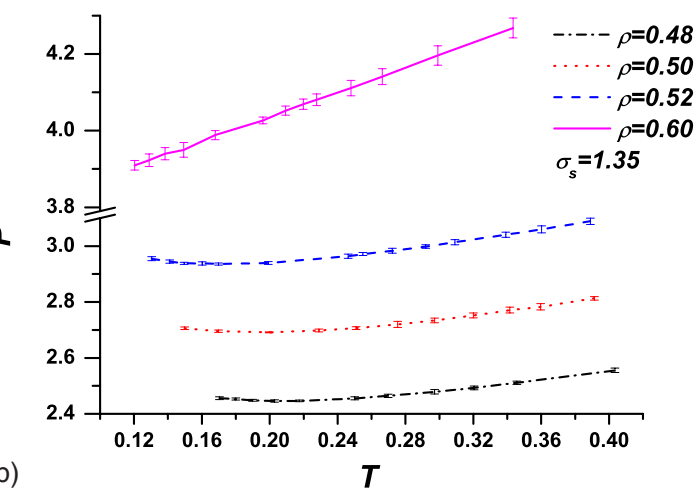

FIG. 7. (Color online) Diffusion anomaly and density anomaly for $\sigma=1.35$.

In order to clarify further the properties of the system in the gap on the phase diagram between the low-density fcc phase and FCO phase, we compute mean-squared displacement $\left[\mathrm{MSD}, \Delta r^{2}(t)\right]$ and self-correlation function $F_{s}(q, t)$ for different temperatures at $\rho=0.53$ (Fig. 4). As usually observed in the proximity of liquid-glass transition, ${ }^{17-19}$ a bending occurs in the MSD after the initial ballistic regime [Fig. 4(a)]. A plateau appears at low temperatures which corresponds to the onset of the caging regime. At long time, the diffusion regime $\left[\Delta r^{2}(t) \propto t\right]$ is reached, when the particles move, on average, a distance of the order of their size.

Another plateau is observed for self-correlation function $F_{s}(q, t)[$ Fig. $4(\mathrm{~b})]$ in the time interval corresponding to the caging regime $[\beta$-relaxation regime in mode-coupling theory $\left.(\mathrm{MCT})^{20-22}\right]$. The correlation functions start to decay from the plateau at times corresponding to the onset of the diffusive regime in the MSD ( $\alpha$-relaxation regime). In order to estimate the transition temperature, we calculate diffusivity $D$ as the long time limit of $\left\langle\Delta r(t)^{2}\right\rangle / 6 t$. As predicted by $\mathrm{MCT}$, in the vicinity of a glass-transition point there is the power-law temperature dependence of the diffusivity $D$ $\propto\left(T-T_{c}\right)^{\gamma}$. In accordance with MCT, ${ }^{20-22} \gamma \geqslant 1.75$. To calculate the parameters of the glass transition, we use the MSD data corresponding to $T=0.12579,0.11933$, $0.10963,0.1084,0.10221,0.09577,0.09342,0.08253$. In this case one has $T_{c}=0.079$ and $\gamma=2.29$. In Fig. 4 we also show the MSD and self-correlation function $F_{s}(q, t)$ corresponding to nonergodic glass regime $(T=0.06916)$.

The apparent glass-transition temperature is above the melting point of the low-density fcc and FCO phases [see
Fig. 2(d)]. This suggests that the "glassy" phase that we observe is thermodynamically stable. This is rather unusual for one-component liquids. In simulations, glassy behavior is usually observed in metastable mixtures (see, e.g., Refs. 17-19) where crystal nucleation is kinetically suppressed. One could argue that, in the glassy region, the present system behaves like a "quasibinary" mixture of spheres with diameters $d$ and $\sigma_{s}$ and that the freezing-point depression is analogous to that expected in a binary system with a eutectic point: there are some values of the diameter ratio such that crystalline structures are strongly unfavorable and the glassy phase is stable even for very low temperatures. The glassy behavior in the reentrant liquid disappears at higher temperatures.

One can expect the frustration to be even more pronounced if we increase the step size. In Fig. 5 we show the phase diagram of the system with the potential (2) for $\sigma_{s}$ $=1.55$. One can see that the system also demonstrates lowand high-density fcc phases separated by fcc to bcc transitions and the amorphous gap which is much more wider than for $\sigma_{s}=1.35$. We did not find any crystal structure between these isostuctural phases in our study. Figures 6(a)-6(c) show the RDFs, mean square displacement, and selfcorrelation functions for $\rho=0.5$. The glass-transition temperature calculated accordingly to MCT is $T_{g}=0.11091$. One can see that the glassification temperature becomes higher. Given the lack of crystal structure between crystalline phases and the increase of the glass-transition temperature, one can assume that the frustration effects become higher with the increase of the step width.

It is also interesting to note that above the low-density fcc phase and in the amorphous gap, the liquid shows some waterlike anomalies for the potential with $\sigma_{s}=1.35$. It is known that for normal liquids, the diffusivity decreases monotonically with increasing density at constant temperature. In contrast, we have observed in our model that for a certain region of densities, $0.4<\rho<0.6$, and for temperatures less than 0.35 , the diffusivity curve has a bend (see upper Fig. 7). This almost coincides with a region in which the isochore has a minimum instead of growing monotonically (see lower Fig. 7). Using the thermodynamic relation $(\partial P / \partial T)_{V}=\alpha_{P} / K_{T}$, where $\alpha_{P}$ is a thermal expansion coefficient and $K_{T}$ is the isothermal compressibility and taking into account that $K_{T}$ is always positive and finite for systems in equilibrium not at a critical point, one can conclude that there is a range of densities and temperatures where the thermal expansion coefficient $\alpha_{P}$ is negative. This kind of anomaly is characteristic, for example, for liquid water.

At low densities, $\rho<0.4$, we have effectively a liquid consisting of spheres with diameter $\sigma_{s}$, at high densities, $\rho$ $>0.6$; the liquid consists of spheres with diameter $d$. In the "anomalous region" in between, our system appears as a mixture of both sorts of particles. This together with the observed anomalies in diffusivity and density further demonstrates that our model shows a quasibinary behavior. Analogous behavior has been demonstrated for a similar model. ${ }^{23}$

The behavior that we observe in this three-dimensional (3D) system bears some striking similarities to that observed in two-dimensional system with "repulsive-shoulder" 


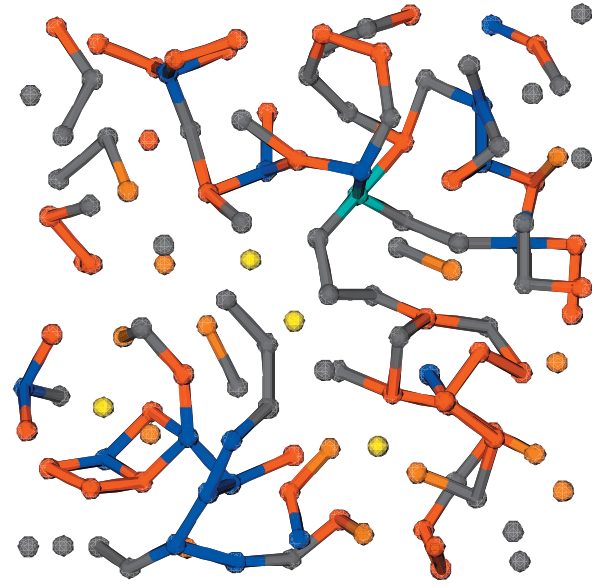

FIG. 8. (Color) Snapshot of the disorderded phase for the $\sigma_{s}=1.35$, for $\rho$ $=0.55, T=0.1$. The colors on the snapshots correspond to the number of the nearest neighbors (yellow, 0; orange, 1; dark gray, 2; red, 3; blue, 4; light blue, 5).

potentials. ${ }^{24-27}$ These simulations suggest that such systems have a low-temperature re-entrant liquid phase where particles intend to arrange in more or less complex, locally ordered patterns. Norizoe and Kawakatsu ${ }^{27}$ found evidence for local clustering and the formation of percolating clusters in the re-entrant liquid phase of a 3D repulsive-shoulder model. Moreover, Refs. 24-27 find that diffusion in this part of the phase diagram is very slow-for this reason, they refer to these percolating cluster phases as "amorphous solids." However, none of these studies includes free-energy calculations and hence the equilibrium phase boundaries between solid and re-entrant liquid are not known. Our free-energy calculations support the notion that these amorphous solids are thermodynamically stable.

In Fig. 8 we represent the snapshot of our system in this region for $\sigma_{s}=1.35$, for $\rho=0.55, T=0.1$. Particles in the snapshot are colored according to the number of the nearest neighbors. The nearest neighbors to the particle $X$ were considered to be all particles within the distance of the first minimum of the radial distribution function. After re-entrant melting the structure corresponds to an assembly of linear "strings" of particles with two near neighbors. It is possible that the structure of liquid here is $3 \mathrm{D}$ analog of the spatial configurations found in Ref. 25. This point clearly requires further study.

In summary, we have performed the extensive computer simulations of the phase behavior of systems described by the soft purely repulsive-step potential (2) in three dimensions. We find a surprisingly complex phase behavior. We argue that the evolution of the phase diagram may be qualitatively understood by considering this one-component system as a quasibinary mixture of large and small spheres. Interestingly, the phase diagram includes two crystalline fcc domains separated by a sequence of the structural phase transitions and a re-entrant liquid that becomes amorphous at low temperatures. The waterlike anomalies were found in the re-entrant liquid for $\sigma_{s}=1.35$.

We thank V. V. Brazhkin for stimulating discussions. The work was supported in part by the Russian Foundation for Basic Research (Grant Nos. 05-02-17280 and 05-02-17621), the Fund of the President of Russian Federation for Support of Young Scientists (MK-2905.2007.2) and NWO-RFBR Grant Nos. 047.016.001. The work of the FOM Institute is part of the research program of FOM and is made possible by financial support from the Netherlands organization for Scientific Research (NWO).

${ }^{1}$ P. C. Hemmer and G. Stell, Phys. Rev. Lett. 24, 1284 (1970); G. Stell and P. C. Hemmer, J. Chem. Phys. 56, 4274 (1972).

${ }^{2}$ New Kinds of Phase Transitions: Transformations in Disordered Substances, Proceedings of NATO Advanced Research Workshop, Volga River, edited by V. V. Brazkhin, S. V. Buldyrev, V. N. Ryzhov, and H. E. Stanley (Kluwer, Dordrecht, 2002).

${ }^{3}$ G. Malescio, J. Phys.: Condens. Matter 19, 07310 (2007).

${ }^{4}$ E. Velasco, L. Mederos, G. Navascues, P. C. Hemmer, and G. Stell, Phys. Rev. Lett. 85, 122 (2000); P. C. Hemmer, E. Velasko, L. Mederos, G. Navascues, and G. Stell, J. Chem. Phys. 114, 2268 (2001).

${ }^{5}$ V. N. Ryzhov and S. M. Stishov, Zh. Éksp. Teor. Fiz. 122, 820 (2002); 95, 710 (2002)]; V. N. Ryzhov and S. M. Stishov, Phys. Rev. E 67, 010201(R) (2003)

${ }^{6}$ Yu. D. Fomin, V. N. Ryzhov, and E. E. Tareyeva, Phys. Rev. E 74, 041201 (2006).

${ }^{7}$ M. R. Sadr-Lahijany, A. Scala, S. V. Buldyrev, and H. E. Stanley, Phys. Rev. Lett. 81, 4895 (1998).

${ }^{8}$ M. R. Sadr-Lahijany, A. Scala, S. V. Buldyrev, and H. E. Stanley, Phys. Rev. E 60, 6714 (1999); P. Kumar, S. V. Buldyrev, F. Sciortino, E. Zaccarelli, and H. E. Stanley, Phys. Rev. E 72, 021501 (2005); L. Xu, S. V. Buldyrev, C. A. Angell, and H. E. Stanley, ibid. 74, 031108 (2006).

${ }^{9}$ E. A. Jagla, J. Chem. Phys. 111, 8980 (1999); E. A. Jagla, Phys. Rev. E 63, 061501 (2001).

${ }^{10}$ D. A. Young and B. J. Alder, Phys. Rev. Lett. 38, 1213 (1977); D. A. Young and B. J. Alder, J. Chem. Phys. 70, 473 (1979).

${ }^{11}$ P. Bolhuis and D. Frenkel, J. Phys.: Condens. Matter 9, 381 (1997).

${ }^{12}$ S. M. Stishov, Philos. Mag. B 82, 1287 (2002).

${ }^{13}$ Daan Frenkel and Berend Smit, Understanding Molecular Simulation: From Algorithms to Applications, 2nd ed. (Academic, New York, 2002).

${ }^{14}$ D. Frenkel and A. J. Ladd, J. Chem. Phys. 81, 3188 (1984).

${ }^{15}$ R. Agrawal and D. A. Kofke, Phys. Rev. Lett. 74, 122 (1995).

${ }^{16}$ C. Rascon, E. Velasco, L. Mederos, and G. Navascues, J. Chem. Phys. 106, 6689 (1997).

${ }^{17}$ W. Kob and H. C. Andersen, Phys. Rev. Lett. 73, 1376 (1994); Phys. Rev. E 51, 4626 (1995); 52, 4134 (1995).

${ }^{18}$ W. Kob, J. Phys.: Condens. Matter 11, R85 (1999).

${ }^{19}$ A. J. Moreno and J. Colmenero, J. Chem. Phys. 125, 164507 (2006).

${ }^{20}$ W. Gotze and L. Sjorgen, Rep. Prog. Phys. 55, 241 (1992).

${ }^{21}$ W. Gotze, J. Phys.: Condens. Matter 11, A1 (1999).

${ }^{22}$ S. P. Das, Rev. Mod. Phys. 76, 785 (2004).

${ }^{23}$ A. B. de Oliveira, P. A. Netz, T. Colla, and M. C. Barbosa, J. Chem. Phys. 125, 124503 (2006); 124, 84505 (2006).

${ }^{24}$ P. J. Camp, Phys. Rev. E 68, 061506 (2003).

${ }^{25}$ G. Malescio and G. Pellicane, Phys. Rev. E 70, 021202 (2004).

${ }^{26}$ M. A. Glaser, G. M. Grason, R. D. Kamien, A. Kosmrlj, C. D. Santangelo, and P. Ziherl, Europhys. Lett. 78, 46004 (2007).

${ }^{27}$ Y. Norizoe and T. Kawakatsu, Europhys. Lett. 72, 583 (2005). 